INTERNATIONAL JOURNAL OF SCIENTIFIC RESEARCH

\title{
ANTERIOR HIP DISLOCATION-AN ORTHOPEDIC EMERGENCY: EVALUATION AND MANAGEMENT OF THESE RARE INJURIES
}

\section{Orthopaedics}

\begin{tabular}{ll}
$\begin{array}{l}\text { Dr Ravindra } \\
\text { Prasad* }\end{array}$ & $\begin{array}{l}\text { Department of Orthopaedics, Rajendra Institute of Medical Sciences, Ranchi, } \\
\text { Jharkhand. }{ }^{*} \text { Corresponding Author }\end{array}$ \\
\hline Dr Rajesh Malwa & Department of Orthopaedics, Rajendra Institute of Medical Sciences, Ranchi, Jharkhand \\
\hline Dr L B Manjhi & Department of Orthopaedics, Rajendra Institute of Medical Sciences, Ranchi, Jharkhand. \\
\hline
\end{tabular}

\section{ABSTRACT}

Anterior hip dislocation with or without associated femoral head fracture or acetabulum are extremely rare injuries. These injuries represent orthopedic emergency of even greater urgency than open fracture and prompt reduction is required to prevent the complications such as AVN and early arthritis. Pathoanatomy and management plan for anterior hip dislocation differ significantly from that of posterior dislocation. When associated with a fracture either of the femoral head or acetabulum, treatment and outcome is significantly changed. A systematic approach is needed to manage these injuries so as to get a congruent and stable hip along with minimizing any complications.

\section{KEYWORDS}

anterior hip dislocation, fracture femoral head, fracture acetabulum

\section{INTRODUCTION}

Pure hip dislocation or dislocation with femoral head fracture are generally the result of high energy trauma, often associated with other systemic injuries. They occur across all age groups and represent a spectrum of injuries that can result when abnormal load is placed on the hip. The majority ( $42 \%$ to $84 \%$ ) occur as the result of a motorvehicle accident. The remainder are associated with falls from a height, sports injuries, and industrial accidents. Posterior dislocations occur much more commonly than anterior dislocations ( $89 \%$ to $92 \%$ ).

The position of the femur when the force is applied determines the pattern of injury. Anterior dislocations are the result of hyperabduction and extension as seen in deceleration injuries. The type of anterior dislocation is determined by degree of hip flexion, with extension leading to superior pubic dislocation and flexion resulting in inferior obturator dislocation. Clinically, there is foreshortening of the involved leg with excessive external rotation and there may be large areas of ecchymosis around abdomen, proximal thigh or knee.

These injuries represent an orthopedic emergency requiring prompt reduction of the hip to protect the femoral head blood supply from further compromise. Additionally, urgent reduction minimizes further stretching of the sciatic nerve. The length of time to relocation has a direct influence on the severity of associated nerve injuries A dislocation of the hip is an emergency of even greater urgency than an open fracture. A standard trauma evaluation, meticulous musculoskeletal and neurologic examination and detailed radiographic assessment is necessary to avoid missing these injuries.

\section{CASE 1}

A 40-year-old male patient was brought into emergency about a week after sustaining trauma due to fall from tree. Physical examination revealed painful right hip with attitude of flexion, abduction and external rotation. Right hip was flexed at 60 degrees, abducted at 30 degrees, fully externally rotated and appeared shortened as compared to opposite normal limb. Extension and adduction were not possible and any attempted movement of hip was extremely painful. No distal neurovascular compromise was found. An immediate anteroposterior radiograph of pelvis including both hip joints was obtained. X ray revealed presence of right obturator type anterior hip dislocation with no associated injuries. Patient was then shifted to operation theatre and Close reduction under general anaesthesia was performed using Walker modification of Allis technique. Here, continuous traction was given in line with femur with slight flexion of hip and then internal rotation and adduction was used to reduce the hip. Reduction was confirmed under fluoroscopy and found to be stable. An immediate $\mathrm{x}$ ray of the pelvis was obtained which showed congruent reduction with no associated fracture. Patient was advised not to bear weight for 8 to 12 weeks. At 6 months follow up, patient did not have any complaint and follow up x-ray did not reveal any avascular necrotic changes or features of arthritis.
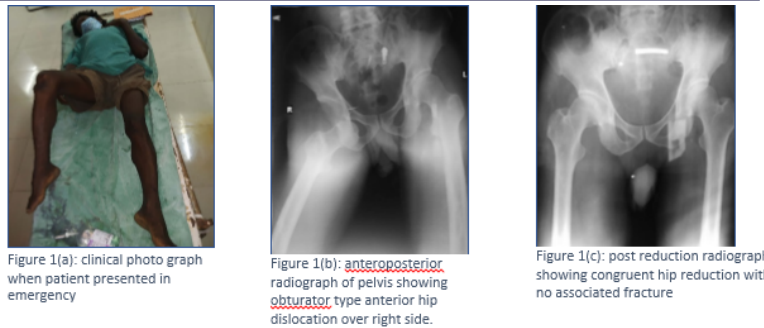

CASE 2

A 50 years old male patient presented to emergency 3 hours after sustaining road accident while driving a motorcycle. Affected left lower limb was markedly externally rotated with ecchymosis around proximal thigh and was kept in flexion and abduction. After assessing for any associated fracture and stabilization of vitals, AP radiograph of pelvis was obtained which showed pubic type of anterior hip dislocation over left side. Patient was immediately scheduled for close reduction under general anaesthesia in OT. Successful reduction was obtained after two attempts and reduction confirmed with fluoroscopy which appeared to be congruent and stable. Post reduction, CT scan of left hip with $2 \mathrm{~mm}$ cuts was obtained to accurately assess joint congruency and to look for any femoral head fracture or intraarticular fragments. CT scan showed congruent hip reduction but with the presence of a shear fracture of inferomedial portion of femoral head which was undisplaced with no intraarticular fragments. As the femoral head fracture was not involving its weight bearing surface and not interfering with the congruency of hip joint, it was planned to manage this injury non operatively. Hence, the same non operative protocol was followed int his case. At follow up of 3 months, there was no limping or restriction of joint motion and radiographic analysis did not reveal any evidence of arthritis or AVN.
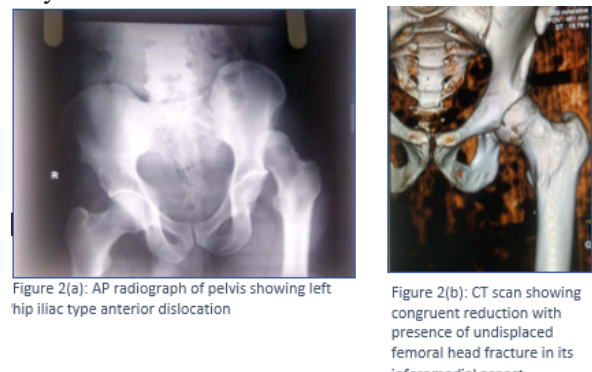

\section{CASE 3}

A 26 years old male suffered injury over left hip while driving a car and was brought to emergency room after 6 hours. There was severe pain over left hip and attitude of the limb was suggestive of an anterior dislocation. After stabilization of vitals and ruling out any associated injuries, immediate AP radiograph of pelvis was obtained which 
confirmed the diagnosis. Emergency close reduction was attempted under GA and after two attempts, we were able to reduce the hip. Fluoroscopy indicated reduced hip but the reduction didn't appear concentric. Subsequently, CT scan of hip was obtained which shown presence of a femoral head fracture over superolateral aspect with an intraarticular free fragment. Traction was applied to involved lower limb and operation scheduled for next day. With the patient positioned lateral, Transtrochanteric approach using the interval between TFL and gluteus maximus with straight trochanteric osteotomy was performed followed by safe surgical dislocation of hip. This approach has the advantages of visualization of entire hip joint allowing treatment of femoral head fracture with or without associated fractures of femoral neck or acetabulum. Intraarticular loose bony fragment was removed from the joint and cartilaginous head fragment was repositioned over superolateral part of head. Postoperatively, active hip motion was deferred for 6 weeks. However, passive hip motion was encouraged with hip musculature strengthening exercises. 6 months follow up of the case revealed excellent result with patient ambulating without pain and performing most of his activities of daily living.
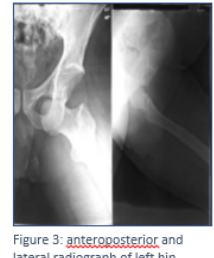

lateral radiograph of left hip showing obturator type anterior

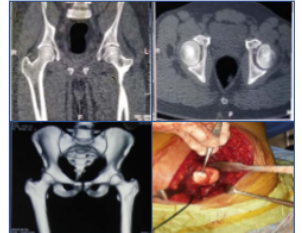

Figure 4: post reduction CT scan showing

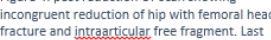

fracture and intraarticular free fragment. Last
intraoperative picture demonstrating femoral

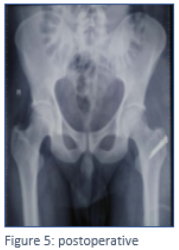

Figure 5: postoperative
radiograph of pelvis

\section{DISCUSSION}

Anterior hip dislocation are much more rare injuries (less than 10\%) than posterior hip dislocation. The leg is held in external rotation, abduction, and mild flexion or extension. The amount of flexion/extension depends on whether the dislocation is superior (pubic) or inferior (obturator). A fullness in the soft tissues in the direction of dislocation may be present.

Evaluation of a patient with suspected hip dislocation should be expedited, beginning with an immediate anteroposterior pelvic radiograph before any attempt at reduction. When evaluating the plain films, first we should look for associated injuries, paying particular attention to the femoral neck, femoral shaft, and acetabulum. Then, congruency of the hip joints should be carefully compared. The head of an anteriorly dislocated hip appears larger on plain radiographs than the contralateral normal hip; a posteriorly dislocated hip appears smaller. A CT scan of the hip should be obtained after reduction to assess the congruency of the hip joint. This assessment is best done by looking for lateral subluxation in the more proximal cuts that show the hip joint and by comparing the joint space in the more distal cuts of the affected hip to that of the uninjured hip. The post reduction CT scan is also the best means for checking for free osteochondral fragments within the joint. MRI can be useful for assessing the hip that has been reduced and has been found to be incongruent but without interposed tissue on CT scan. The MR image is better at evaluating the labrum, the muscles, and the capsule that may be incarcerated within the joint. MRI may also show damage to the obturator externus muscle, which may represent injury to the medial circumflex femoral artery and possibly an increased risk of avascular necrosis.

Associated injuries fall into two categories: those associated with the dislocation and those associated with the precipitating trauma. Additional organ system injuries occur in $95 \%$ of patients with a traumatic hip dislocation secondary to a motor vehicle crash; $33 \%$ of patients have other orthopedic injuries, $15 \%$ have abdominal injuries, $24 \%$ have closed-head injuries, $21 \%$ have thoracic injuries, and $21 \%$ have craniofacial injuries. A higher percentage of anterior dislocations have an associated femoral head fracture $(68 \%)$ as compared to posterior dislocations $(7 \%)$. The incidence of femoral head fractures is higher with anterior dislocations because the strong anterior ligaments do not easily allow for subluxation of the hip. The anterior wall of the acetabulum is substantial and resists fracturing more than the posterior wall, so the femoral head becomes the weak link and is fractured by the shear force.

Incidence of $\mathrm{AVN}$ is rare with anterior dislocation as it relaxes the medial femoral circumflex artery, the chief vessel supplying the femoral head whereas Posterior dislocations puts this vessel at risk explaining the $2 \%$ to $17 \%$ rate of $\mathrm{AVN}$ with posterior dislocations. Anterior hip dislocations are classified by their anatomic location, being superior, including pubic or subspinous, or inferior, including obturator, thyroid, and perineal locations. In anterior dislocations, femoral head impaction injuries are more common than shearing injuries which are more apparent on CT scans. Epstein classified anterior dislocation based on direction of dislocation, with or without associated fracture of the femoral head or acetabulum. Brumback et al suggested a unified classification used for both anterior and posterior hip dislocations. It takes into account the size of the femoral head fragment, the direction of dislocation as well as the stability of the hip. In this classification, anterior hip dislocations fall either in type 3 or type 4 . In type 3, dislocation is associated with a femoral neck fracture and in type 4, anterior dislocations are classified as Indentation or Transchondral type. Regardless of the classification system used, important elements are whether there is an associated fracture and whether the hip is stable after reduction.

\section{Table 1: Epstein classification of anterior hip dislocation}

\begin{tabular}{|c|c|}
\hline Type & Description \\
\hline Type 1 & Superior dislocations, including pubic and sub spinous \\
\hline 1A & No associated fractures \\
\hline 1B & Associated fracture or impaction of the femoral head \\
\hline 1C & Associated fracture of the acetabulum \\
\hline Type 2 & Inferior dislocations, including obturator and perineal \\
\hline 2A & No associated fractures \\
\hline 2B & Associated fracture or impaction of the femoral head \\
\hline 2C & Associated fracture of the acetabulum \\
\hline
\end{tabular}

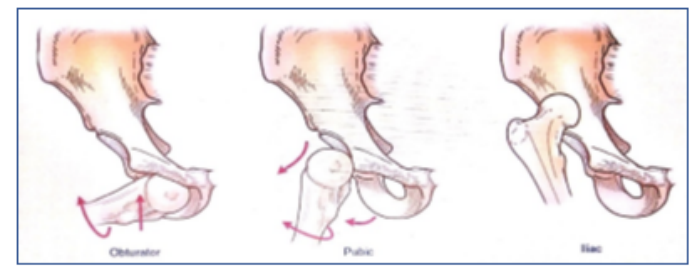

Figure 6: Types of anterior dislocation based on anatomic location

Closed Reduction: For Inferior dislocation, Walker modification of Allis technique is used. Traction is continuously applied in line of femur with gentle flexion. Along with a lateralizing force on proximal femur, internal rotation and adduction are used to reduce the hip. In case of superior dislocation, distal traction is applied until the head is at the level of the acetabulum and the gentle internal rotation is applied. Continuous traction should be used rather than short jerky motions. Following reduction, stability of the hip is assessed. If the reduced hip is grossly unstable, skeletal traction should be applied. Following reduction, immediate radiograph of pelvis is obtained. If the hip is not reduced, another reduction is performed, preferably in the operating room. If the hip is reduced, a CT scan is obtained to assess the congruency and look for fragments. The joint space on the involved hip should be identical to that on the uninvolved hip.

Post reduction assessment: Following reduction, either of these three conditions may be encountered

1. Congruent reduction without associated fracture

2. Congruent reduction with associated fracture of the femoral head: treatment depends upon the size and reduction of head fragment. Small fragment may be treated nonoperatively regardless of its position. However, it this small fragment is caudal, it may be excised. Larger fragments affecting the weight baring surface has to be reduced and fixed with low profile or headless compression screws. Surgical dislocation of hip has become the preferred method to approach fracture dislocation of hip when there is involvement of the femoral head and has been found to improve clinical outcome compared to more traditional anterior and posterior approaches.

\section{Incongruent reduction which requires open reduction}

Open Reduction: Inability to obtain a closed reduction is usually the result of inadequate relaxation/paralysis, blockage by the hip capsule 
and/or short external rotators, or a femoral fracture that makes control of the hip difficult. Failure to obtain a closed reduction is an indication for an urgent and immediate open reduction. In addition, hips with associated fractures that are unstable after reduction, injury to sciatic nerve during reduction attempt and hips that are not congruent after reduction all need open treatment. An anterior approach via a SmithPetersen or Watson-Jones approach should be chosen.

Post reduction management: Weightbearing status is dictated by time of reduction since injury as well as associated fractures if any are present. For patients whose hip has been reduced within 6 hours and in pure dislocation, brief period of rest for several days to 2 weeks is recommended followed by mobilization. Early weightbearing does not predispose to the development of AVN as previously contended Extremes of motion are avoided for 6 to 8 weeks to allow for capsular healing. Most of these patients can achieve full weight bearing by 6 weeks. In case, if dislocation is associated with fracture and in hips reduced after 6 hours, full weight bearing is delayed for 8 to 12 weeks.

\section{CONCLUSION}

Dislocation of hip whether anterior or posterior, represents an orthopedic emergency of even greater urgency than an open fracture. It requires prompt reduction of the hip to protect the femoral head blood supply from further compromise. Additionally, urgent reduction minimizes further stretching of the sciatic nerve. The length of time to relocation has a direct influence on the severity of associated nerve injuries. The rates of AVN and early arthritis are increased if the hip is left dislocated for more than 6 hours. Pathoanatomy and management plan for anterior hip dislocation differ significantly from that of posterior dislocation. Anterior hip dislocation associated with a fracture either of the femoral head or acetabulum significantly changes the treatment and outcome of hip dislocation. Use of Transtrochanteric or surgical dislocation approach for these injuries has shown improved clinical outcomes as compared to more traditional anterior and posterior approaches. Regardless of the direction of dislocation, our approach should be to avoid complication by emergent reduction and to obtain a congruent and stable hip.

\section{Reference}

1. Kain MS, Tornetta Paul. Hip dislocation and fractures of the femoral head. Rockwood and Greens Fracture in Adults. 8th ed. 2015; (1): 1983-2024

2. CW John. Fracture and dislocations of the hip. Campbells Operative Orthopedics. 13th ed. $2017 ;(2): 2852-2855$

3. CL Steven, AO Steven. Hip dislocations and fracture of the femoral head. Review of Orthopedic Trauma. 2nd ed. 2013; (1): 654-697

R Shanmugnathan, D Jayaramaraju. Dislocations and fracture dislocation of the hip. Mercer's textbook of Orthopedics and Trauma. 10th ed. 2012;(1):338

5. WH Gregory. Hip. Simon's Emergency Orthopedics. 7th ed. 2015; (1): 437

6. AC Tekin, H Cabuk, CD Buyukkurt, SS Dedeoglu, Y İmren, and H Gurbuz, "Inferior hip dislocation after falling from height: a case report," International Journal of Surgery Case Reports, vol. 22, pp. 62-65,2016

7. AM Phillips and A Konchwalla, "The pathologic features and mechanism of traumatic dislocation of the hip," Clinical Orthopaedics, vol. 377, no. 1, pp. 7-10,2000

8. P Kellam and RF Ostrum, "Systematic review and meta-analysis of avascular necrosis and posttraumatic arthritis after traumatic hip dislocation," Journal of Orthopedic Trauma, vol. 30 , no. 1 , pp. $10-16,2016$

9. MJ Gardner, M Suk, A Pearle, RL Buly, DL Helfet, and DG Lorich, "Surgical dislocation of the hip for fractures of the femoral head," Journal of Orthopaedic Trauma, vol. 19, no. 5,pp. 334-342,2005

10. MF Mostafa, W El-Adl, and MA El-Sayed, "Operative treatment of displaced Pipkin type I and II femoral head fractures," Archives of Orthopaedic and Trauma Surgery, vol. 134, no. 5, pp. 637-644, 2014

11. JP Stannard, HW Harris, DA Volgas, and JE Alonso, "Functional outcome of patients with femoral head fractures associated with hip dislocations," Clinical Orthopaedics and Related Research, vol. 377, pp. 44-56, 2000

12. Phillips AM, Konchwalla A. The pathologic features and mechanism of traumatic dislocation of the hip. Clin Orthop Relat Res. 2000;(377):7-10.

13. Toms AD, Williams S, White SH. Obturator dislocation of the hip. J Bone Joint Surg. 2001;83(1):113-5 